\title{
Energy Efficient Distributed Image Compression in Resource Constrained Multihop Wireless Networks
}

\author{
Huaming $\mathrm{Wu}$ and Alhussein A. Abouzeid \\ Department of Electrical, Computer and Systems Engineering \\ Rensselaer Polytechnic Institute, Troy, New York 12180 \\ Email:wuhm@rpi.edu, abouzeid@ecse.rpi.edu
}

\begin{abstract}
Efficient compression and transmission of images in a resource constrained multihop wireless network is considered. Distributed image compression is proposed as a means to overcome the computation and/or power limitation of individual nodes by sharing the processing of tasks. It has the additional benefit of extending the overall lifetime of the network by distributing the computation load among otherwise idle processors. Two design alternatives for energy efficient distributed image compression are proposed and investigated with respect to energy consumption and image quality. Simulation results show that the proposed scheme prolongs the system lifetime at a normalized total energy consumption comparable to centralized image compression.
\end{abstract}

\section{INTRODUCTION}

Advances in visual sensors [1], [2] and wireless communication have enabled the development of low-cost, low-power visual multihop wireless networks, which have recently emerged for a variety of applications, including environmental and habitat monitoring, target tracking and surveillance [3], [4]. However, representing visual data requires a large amount of information, leading to high data rates, which in turn requires high computation and communication energy. Consequently, visual sensor networks present several challenges beyond those common to low data rate sensing, such as acoustics, temperature or pressure.

We consider compressing and transmitting images in a multihop wireless network. We focus on the design and performance evaluation of distributed image compression algorithms. Distributed image compression in sensor networks is beneficial for two common scenarios. One scenario is when nodes have extremely constrained computation power. Hence, any single node may not have sufficient computation power to completely compress a large raw image. In this case, a distributed method to share the processing task is necessary. Another scenario is that even when nodes are not extremely computation power constrained, but are battery operated, distributing the computation load among otherwise idle processors of other nodes extends the overall lifetime of the network.

In this research, we have made a first attempt at the design and performance evaluation of distributed image compression in energy constrained multihop wireless (e.g. ad hoc or sensor) networks. By exploiting the characteristics of the Discrete Wavelet Transform (DWT), we propose a distributed image compression scheme where nodes compress an image while forwarding it to the destination subject to a specific image quality constraint. In particular, we propose two data exchange methods of distributed wavelet transform and investigate their performance in terms of energy consumption and image quality over a multihop wireless network. Simulation results show that our scheme prolongs the system lifetime by up to 4 times and has a total energy consumption comparable to the centralized algorithm.

In Section II, we examine related work. The system model is described in Section III. Section IV introduces the distributed image compression schemes. Simulations of the proposed schemes are presented in Section V. We conclude the paper in Section VI.

\section{RELATED WORK}

Up to our knowledge, distributed image compression in wireless ad hoc networks has not been studied in the literature. However, our work has been inspired by a variety of other research efforts. We describe below some of the ideas and basic concepts that inspired our work, and reflect on how our work relates to current research in the area of sensor network applications.

While early research efforts in wireless sensor networks did not investigate the issues of node collaboration, focusing more on issues in the design and packaging of small, wireless devices [5], [6], more recent efforts (e.g. [7], [8]) have considered node collaboration issues such as data "aggregation" or "fusion". Our approach of distributed image compression falls within the domain of techniques that apply the concept of in-network processing, i.e. processing in the network by computing over the data as it flows through the nodes. It is worth noting that current aggregation functions (e.g., "maximum" and "average" [7]) are limited to scalar data. Our approach can be viewed as an extension to vector data aggregation. Previous distributed signal processing/compression problems (e.g. [9], [10]) exploit correlations between data at close-by sensors in order to jointly compress or fuse the correlated information resulting in savings in communication energy. Fusion of correlated images is not discussed in this paper.

In parallel distributed computing theory [11], a problem (or task) is divided into multiple sub-problems (or sub-tasks) of smaller size (in terms of resource requirements). Every node solves each subproblem by running the same local algorithm, 
and the solution to the original problem is obtained by combining the outputs from the different nodes. Our approach to the design of distributed image compression is similar in concept, in that we distribute the task of image encoding/compression to multiple smaller image encoding/compression sub-tasks. However, a key difference is that distributed computation theory typically focuses on maximizing the speed of execution of the task while our primarily concern here is reducing the total energy consumption subject to a required image quality.

Thus, our proposed approach of image compression intersects with the literature on parallel wavelet transform, which primarily focuses on parallel DWT on special purpose hardware (e.g., application specific VLSI architectures [12], FPGAs [13], DSPs [14]) or general purpose multiprocessor systems [15], [16]. The difference, of course, is that previous parallel wavelet transform research assumes the energy cost of data movement between processors to be zero or very small compared to the wavelet transform computation. This assumption is no longer valid in multihop wireless networks due to costly wireless communication among nodes. Furthermore, the main objective of previous parallel wavelet transform is to speedup the transform. Wireless nodes operating on battery power will possibly have their primary objective as the conservation of their energy reserves rather than algorithm run time.

Distributed Fast Fourier Transform (FFT) in a sensor network was considered in [17]. Our work on DWT is similar in spirit, in the sense that FFT and DWT are two signal processing techniques. However, our problem has two additional dimensions. First, DWT is just one component of the image compression and transmission problem, and in that sense our problem includes more complex network-wide challenges. Second, while the spectrum of possible outcomes of an FFT is limited, in the sense that the FFT is either successfully computed or not, the image encoding and transmission problem offers a wide continuum of solutions in terms of the received image quality.

Recently, applications of classical image processing techniques in sensor networks have gained a lot of interest for the parallels between image processing and sensor networks. By mapping individual sensors as pixels in an image, [19] examined cleaning of uncorrelated sensor noise, and the decentralized detection of edges. Ganesan et al. [20] have proposed a generalized hierarchical architecture for multiresolution querying of regularly placed sensor networks that is based on wavelet transforms. Servetto [21] also exploits wavelet transforms to decorrelate sensor data to address the sensor broadcast problem where every sensor observes only one pixel. These works focus on the correlation between sensor nodes. In this paper, we consider nodes observing an image instead of a pixel and investigate the problem of compressing and transmitting images in sensor networks.

A comment regarding our previous work on image compression in sensor networks is in order. Traditionally, maximal image compression (source coding) is implemented at the source in order to reduce the number of bits transmitted thus reducing the communication energy. The goal of our previous work [18] is to minimize the overall energy consumption of the network by computing the optimal compression parameters as a function of the network density. (It turns out that maximal compression at the source is not always optimal). Centralized image compression was used at the source nodes which may limit the lifetime of the network. Distributed image compression was not discussed in [18].

Finally, we believe it is appropriate to also mention some of the projects related to visual sensor networks. In [22], a data centric routing protocol is proposed for efficient image retrieval in wireless ad hoc networks by using metadata of images. The issue of the transmission/encoding of the image, once located, is not studied. A topology control approach to maximize the network lifetime of a wireless video surveillance network by arranging nodes location is presented in [23].

\section{SySTEM MODEL}

We consider a densely deployed multihop wireless network in which some of the nodes are camera-equipped. Every camera-equipped node can respond to an image query by generating a raw image (e.g. a snapshot of its sensing area in the case of a sensor network) and transmitting this image to the destination (sink). When sending an image request (query), the destination node specifies the desired image quality. A cluster based routing mechanism is assumed to be in place such that nodes can self-organize into a two-tiered cluster (e.g. [24]). In this initial investigation, the communication environment is assumed to be contention-free. A session refers to a source sending one image to a destination, in response to receiving a request from the destination. It is worth noting that there are typically multiple sessions at a time.

For this study, we use a transceiver energy dissipation model similar to the one proposed in [25]. The energy consumed in transmission per bit is

$$
E_{T X}=\epsilon_{e}+\epsilon_{a} d^{\alpha}
$$

and the energy consumed in reception per bit is

$$
E_{R X}=\epsilon_{e}
$$

where $\epsilon_{a}$ is the energy dissipated per bit per $m^{2}, \epsilon_{e}$ is energy consumed by the circuit per bit, $d$ is the distance between a wireless transmitter and a receiver, and $2 \leq \alpha \leq 4$ is the path loss parameter [26]. The energy consumed in computation per bit is

$$
E_{D W T}=\gamma
$$

where $\gamma$ is the energy dissipated for one level wavelet transform compression (described in Section IV-A) per bit. The energy spent in quantization and entropy coding per bit is ${ }^{1}$

$$
E_{E N T}=\delta \text {. }
$$

The performance metric in this paper is system lifetime, which is defined as the time duration from the time instant

\footnotetext{
${ }^{1}$ Section $\mathrm{V}$ describes how $\gamma$ and $\delta$ are estimated.
} 
when the network starts working until the first node in the network fails due to depleted energy.

Image quality is measured using the Peak Signal-To-Noise Ratio (PSNR) metric, which is defined (in decibels) as

$$
P S N R=20 \log _{10} \frac{2^{b}-1}{E\|x(i, j)-\hat{x}(i, j)\|}
$$

where $x(i, j)(\hat{x}(i, j))$ are the pixel values of the original (reconstructed) image, and $b$ is the number of bits per pixel (bpp) of the original image. We recognize that the PSNR does not always accurately model perceptual image quality, but we use it because it is a commonly used metric in the literature. In addition, we also show a few examples on a test image to show certain aspects of the received image that are not revealed by the PSNR value.

\section{Distributed IMAge COMPREsSion}

Since we are investigating the problem of transmitting images in multihop wireless networks, it is beneficial to start by describing some of the background of image compression as it relates to this paper. Then we describe our approach of distributed image compression.

\section{A. Background on Image Compression}

A common characteristic of most images is that the neighboring pixels are correlated and therefore contain redundant information. Image compression aims at reducing the number of bits needed to represent an image by removing the spatial and spectral redundancies as much as possible. Among a variety of image compression algorithms, JPEG [28] is a commonly used standard for still image compression. More recently, the wavelet transform has gained widespread acceptance in signal processing in general, and in image compression research in particular. In many applications, wavelet-based schemes (also referred to as subband coding) outperform other coding schemes. Wavelet-based coding is more robust under transmission and decoding errors, and also facilitates progressive transmission of images. Because of their inherent multi-resolution nature [29], wavelet coding schemes are especially suitable for applications where scalability and tolerable degradation are important. Thus, we choose the new wavelet-based image compression standard JPEG2000 [30] as the image compression scheme in this study.

Wavelet transform coding first transforms the image from its spatial domain representation to a different type of representation using wavelet transform and then codes the transformed values (coefficients) [31]. A typical wavelet-based image compression system is shown in Fig. 1. It consists of three components; Forward/Reverse Wavelet Transform, Quantizer/Dequantizer and Entropy Encoder/Decoder. In terms of energy dissipation of JPEG2000 compression/decompression, wavelet transform is the dominant part [32].

The fundamental concept behind wavelet transform is to split up the frequency band of a signal (image in our case) and then to code each subband using a coder and bit rate accurately matched to the statistics of the band. There are

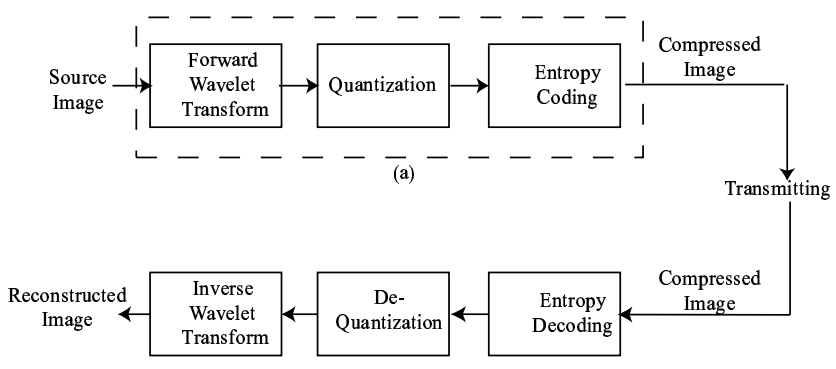

(b)

Fig. 1. A typical wavelet-based image compression (a)encoder (b)decoder.

several ways wavelet transforms can decompose a signal into various sub-bands. These include uniform decomposition, octave-band decomposition, and adaptive or wavelet-packet decomposition [33]. Out of these, octave-band decomposition is the most common, and hence is used in this paper. This is a non-uniform band splitting method that decomposes the lower frequency part into narrower bands and the high-pass output at each level is left without any further decomposition.

The octave-band decomposition procedure can be described as follows. A Low Pass Filter (LPF) and a High Pass Filter (HPF) are chosen, such that they exactly halve the frequency range of the input signal. First, the LPF is applied for each row of data, thereby getting the low frequency components of the row. When viewed in the frequency domain, the output data contains frequencies only in the first half of the original frequency range because the LPF is a half band filter. Therefore, the output of the LPF can be sub-sampled by 2 according to Shannon's sampling theorem, in which case the output data will contain only half the original number of samples. Then, the HPF is applied for the same row of data, and similarly the high pass components are separated. The low and high pass components are arranged into a row of output data as illustrated in Fig. 2(a). This procedure is performed on all rows, which we term "1-D wavelet transform." Next, the filtering is done for each column of the intermediate output data. This whole procedure (including both row and column operations) is called a "2-D wavelet transform." The resulting two-dimensional array of coefficients, depicted in Fig. 2(b), contains four bands of data, each labeled as LL (low-low), HL (high-low), LH (low-high) and HH (high-high). The LL band can be further decomposed in the same manner, thereby producing even more sub-bands as shown in Fig. 2(c). This can be repeated up to any level, thereby resulting in a pyramidal decomposition as depicted in Fig. 2.

Following the wavelet transform operation, bit allocation [34], quantization and entropy coding [35] are applied to get the final compressed image.

\section{B. Distributed Wavelet Transform}

The basic idea of the proposed distributed image compression is distributing the workload of wavelet transform to several groups of nodes along the path from the source to the destination. The key issue in the design of distributed wavelet- 


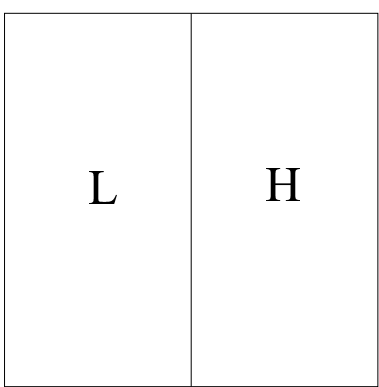

(a) 1-D wavelet transform

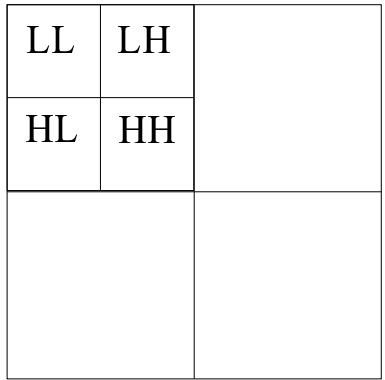

(c) Two level decomposition

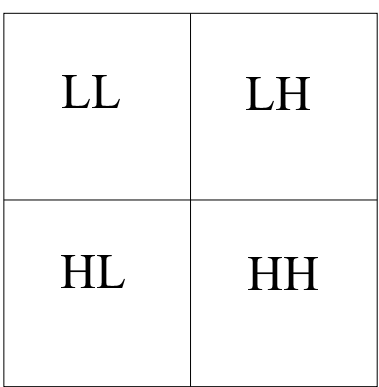

(b) Single level decomposition

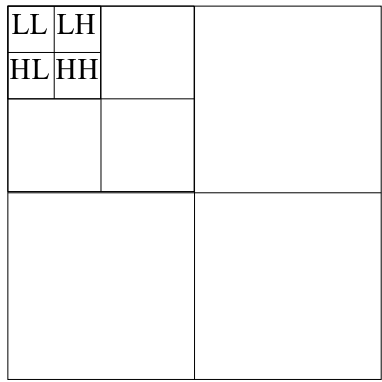

(d) Three level decomposition
Fig. 2. Illustration of wavelet spectral decomposition and ordering. L stands for low frequency component and $\mathrm{H}$ stands for high frequency component.

based image compression is data exchange (e.g. exchange of raw image or intermediate results). The importance stems from the wireless communication energy that is incurred by different data exchange schemes. In the research of parallel wavelet transform, data is broadcasted to all processors to speedup the execution time [36] which may increase the energy consumption. We proposed two data exchange schemes and compare between them with respect to image quality and energy consumption; 1)Method 1: Divide by rows/columns; 2)Method 2: Tiling.

1) Method 1: In this method, we consider the data partitioning scheme proposed in traditional parallel wavelet transform [36] when applied to a multihop wireless network. Fig. 3 illustrates the data partitioning scheme for one wavelet decomposition level. At first, the data is partitioned into $n$ blocks $R_{1}, R_{2}, \ldots, R_{n}$ where each block consists of one or more rows. Then, each node runs 1-D wavelet transform on $R_{i}$. Once the 1-D wavelet transform is completed on all rows, one node collects the intermediate results $Q_{1}, Q_{2}, \ldots, Q_{n}$ and divides the results into $m$ blocks $I_{1}, I_{2}, \ldots, I_{m}$. Then each node applies 1-D wavelet transform on $I_{i}$. Finally, a node gathers the 2-D wavelet transform results $J_{1}, J_{2}, \ldots, J_{m}$. This data exchange scheme does not result in any image quality loss compared to the traditional centralized scheme.

An example of distributed image compression using four nodes in each cluster is shown in Fig. 4. After receiving a query from a source node $s$, the cluster head $c_{1}$ selects a set of nodes $P_{1}$ in the cluster which will take part in the distributed wavelet transform then informs $s$. $s$ divides the raw image data by rows and transmits them to $P_{1}\left(p_{11}, p_{12}, p_{13}\right.$ and $\left.p_{14}\right)$. Those nodes run $1-\mathrm{D}$ wavelet transform algorithm on

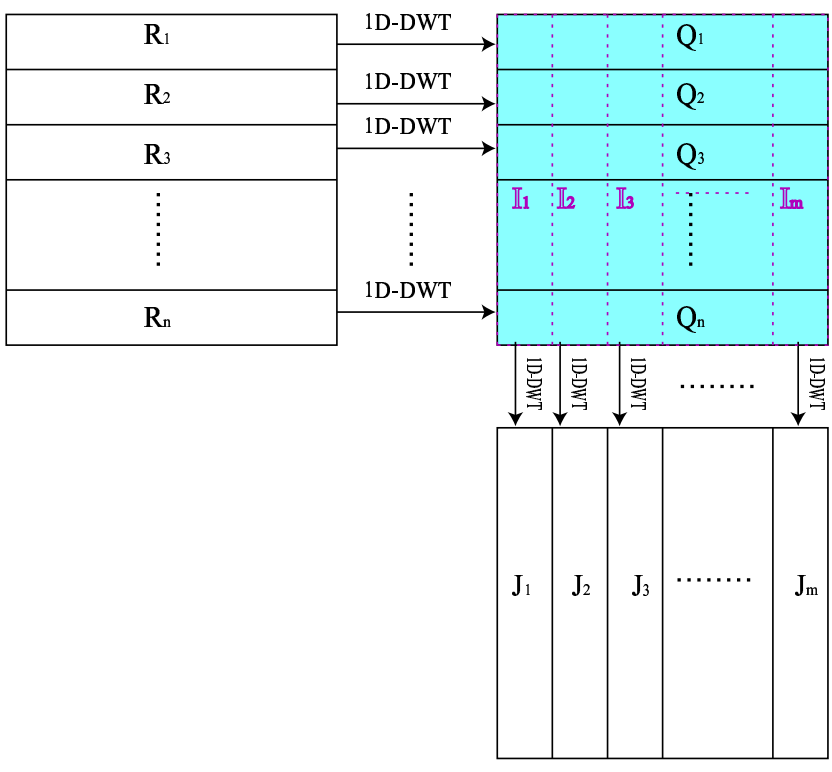

Fig. 3. Traditional data partitioning approach: divide by rows/columns for one wavelet decomposition level. The set of $R_{i}(i=1,2, \ldots, n)$ is the data to be wavelet transformed. The set of $J_{i}(i=1,2, \ldots, m)$ is the 2-D wavelet transform results.

their received data then send the intermediate results back to $c_{1} . c_{1}$ combines the results and divides data by columns to $P_{1}$ again. These nodes process data and send the results (Level 1 data in Fig. 4) to the next cluster head $c_{2}$. After receiving the results, $c_{2}$ chooses a part of the results (corresponding to LL in Fig. 2(b)) and distributes it to the set of nodes $P_{2}$ $\left(p_{21}, p_{22}, p_{23}\right.$ and $\left.p_{24}\right) . c_{2}$ will code and send the other part of the received data (corresponding to $\mathrm{LH}, \mathrm{HL}$ and $\mathrm{HH}$ in Fig. 2(b)) directly to the next cluster head $c_{3}$. The nodes in $P_{2}\left(p_{21}, p_{22}, p_{23}\right.$ and $\left.p_{24}\right)$ will also send their processed results (corresponding to Level 2 wavelet decomposition as in Fig. 2(c)) to $c_{3}$ after running 1-D wavelet transform twice. Depending on the image quality specified by the query (which is application-dependent), this procedure may continue on $c_{3}$ and its following nodes until the final compressed image reaches the destination (sink) node. It should be noted that, as shown in Fig. 4, the cluster heads $\left(c_{1}, c_{2}\right.$ and $\left.c_{3}\right)$ have to collect and transmit the intermediate 1-D wavelet transform results (indicated by two-way arrows) during each wavelet decomposition level.

2) Method 2: Tiling, which is used in JPEG [28], can also be used in wavelet based image compression. Normally, the wavelet decomposition is computed on the entire image, which inhibits annoying compression blocking artifacts that occur at low bit rate coding. However, JPEG2000 [30] also supports the concept of image tiling for operation in low memory environments. In this case, the image is partitioned into tiles (blocks), and then each block is sent to a node to do 2-D wavelet transform independently. Once a node completes its 2-D wavelet transform, it sends the result back to a center node. The processing of tiles independently leads to a rate-distortion loss and blocking artifacts as the number 


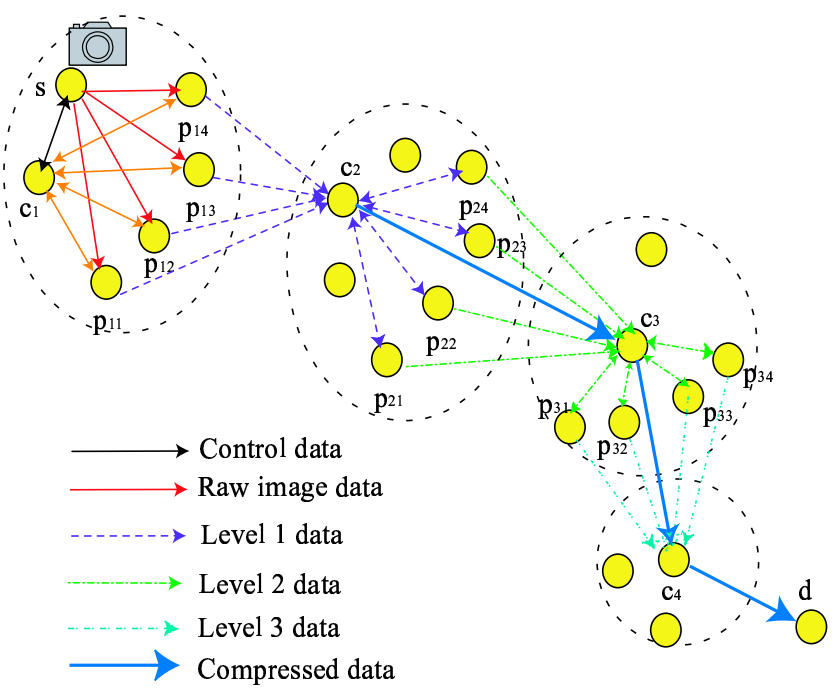

Fig. 4. Illustration of data exchange Method 1 of distributed wavelet-based image compression in a multihop wireless network. Three levels of wavelet decomposition. $s$ is the source node. $d$ is the destination (sink) node. $c_{i}$ is the cluster head of cluster $i$. Two-way arrows between cluster heads and nodes indicate that the cluster heads collect and transmit the 1-D wavelet transform results during each wavelet decomposition level.

of tiles and/or processors is increased [16]. We found that the quality loss and blocking artifacts are small when the number of tiles is small or when the bit rate of compressed image is not very low. Fig. 5 illustrates the image quality degradation of tiling. It is observed that the quality loss of tiling with four tiles (Fig. 5(c)) compared to the result without tiling (Fig. 5(a)) is smaller than $0.2 d B$ in terms of PSNR. The blocking artifacts are also negligible with a moderate bit rate (Fig. 5(d)). Therefore, it is still applicable in distributed wavelet-based image compression if the number of tiles is small or for not very low bit rate. Post processing techniques can also be applied at the destination to cope with the blocking artifacts [37].

Fig. 6 shows an example of distributed image compression using four tiles and three wavelet decomposition levels. Similar to Method 1, after receiving a query from a source node $s$, the cluster head $c_{1}$ selects a set of nodes $P_{1}$ in the cluster which will take part in the distributed wavelet transform then informs $s$. $s$ divides the raw image data into tiles and transmits them to $P_{1}$. Those nodes run 2-D wavelet transform on their received image data then send the results (Level 1 data in Fig. 6) individually to the next cluster head $c_{2}$. For each tile, $c_{2}$ chooses a part of the results (corresponding to LL in Fig. 2(b)) and sends it to one of the processing nodes $\left(p_{21}, p_{22}, p_{23}\right.$ and $\left.p_{24}\right) . c_{2}$ will also combine the other parts of the received data (corresponding to LH, HL and HH in Fig. 2(b)) from all tiles then code and send the result directly to the next cluster head $c_{3}$. In this way, the final compressed image reaches the destination node.

\section{Quantization and Coding}

The bit allocation, quantization step and entropy coding method in the proposed distributed image compression use the

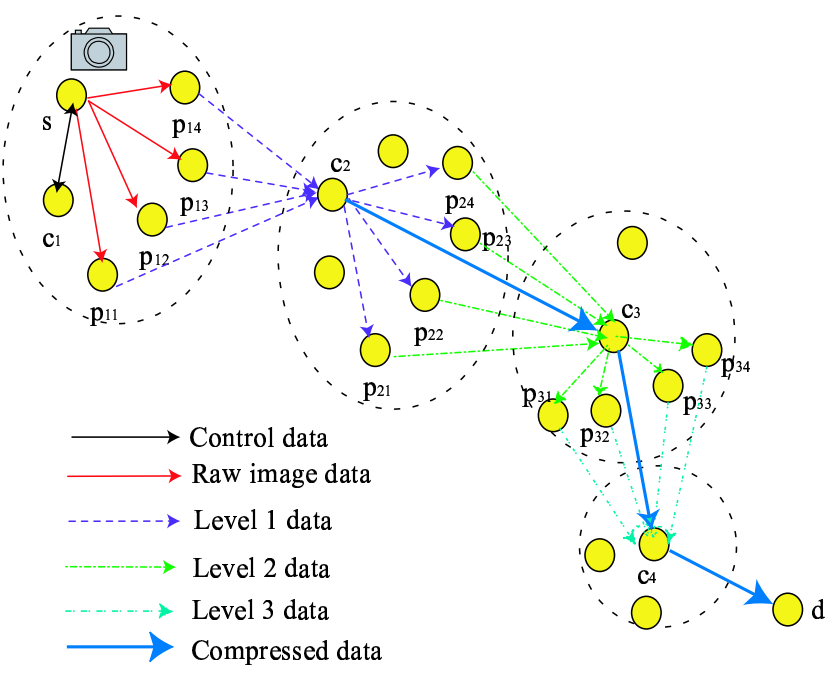

Fig. 6. Illustration of data exchange Method 2 of distributed waveletbased image compression in a wireless multihop network. Three levels of decomposition. $s$ is the source node. $d$ is the destination node. $c_{i}$ is the cluster head of cluster $i$.

same method as in the centralized JPEG2000 image compression. In the Embedded Block Coding method which is used in JPEG2000 standard [38], each subband (corresponding to LL, $\mathrm{LH}, \mathrm{HL}$ and $\mathrm{HH}$ component at each wavelet decomposition level) is divided into small blocks called "code blocks." And then each code block ${ }^{2}$ is coded independently. More details of Embedded Block Coding in JPEG2000 can be found in [30], [39]. It is worth noting that the nodes will compress the coefficients which do not need further wavelet transform at the target bit rate. For instance, those coefficients corresponding to $\mathrm{LH}, \mathrm{HL}$ and $\mathrm{HH}$ components at each decomposition level will be compressed to the target bit rate.

It is worth noting that, in the proposed distributed wavelet transform, nodes run entropy coding before exchanging data (whether raw image pixels or transformed LL coefficients) to save communication energy. This is in contrast to the centralized or traditional parallel version of wavelet transform, where data exchange occurs without any entropy coding. The reason is that traditional approaches are not concerned with the communication energy cost and thus the entropy coding is applied only once, after the required level of wavelet decomposition is reached (as depicted in Fig. 1).

\section{Performance Evaluation}

In this section, we perform two sets of simulations that compare our proposed distributed image compression with the centralized algorithm based on two performance metrics: total energy consumption and system lifetime.

\section{A. Simulations and Experiments Setup}

This section presents the simulation scenarios for the evaluation of the performance of the proposed protocols, the

\footnotetext{
${ }^{2}$ The size of code blocks is a flexible parameter. A typical size of the code block is 64 by 64 .
} 


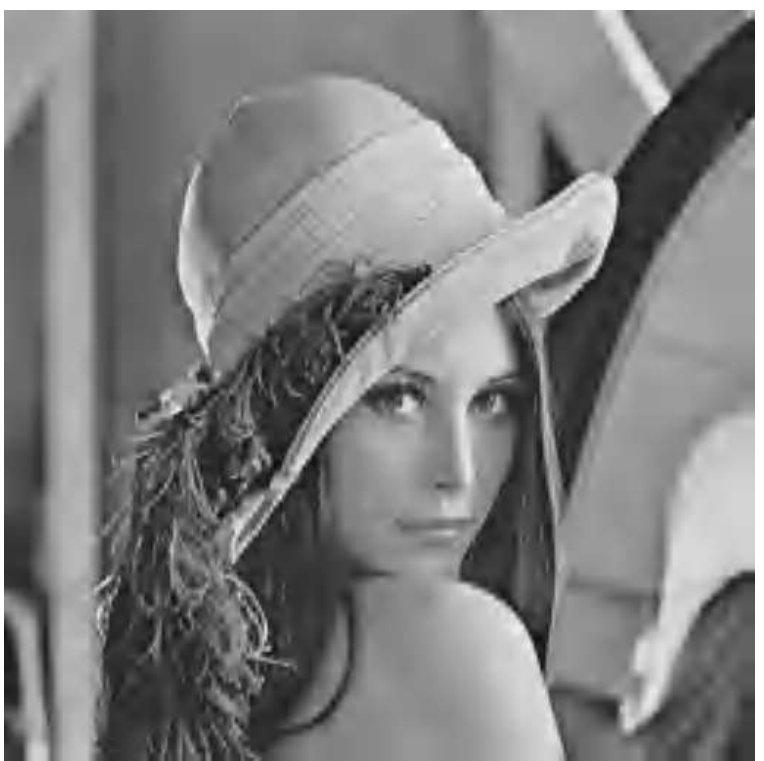

(a) JPEG2000 without tiling. Bit rate is 0.1bpp. PSNR= 29.30dB.

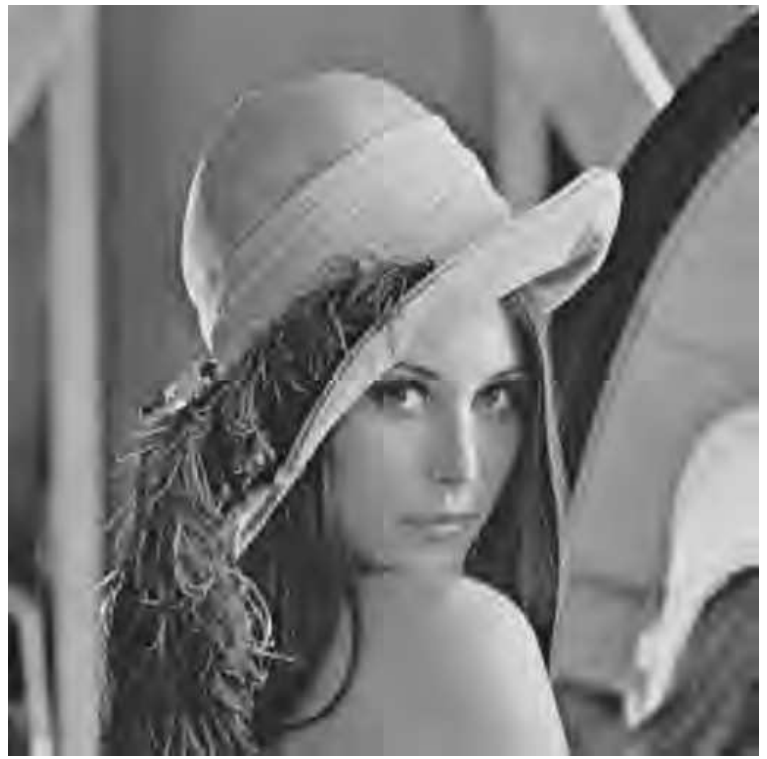

(c) JPEG2000 with a tile size $256 \times 256$ pixels. Bit rate is $0.1 b p p$. $P S N R=29.12 d B$.

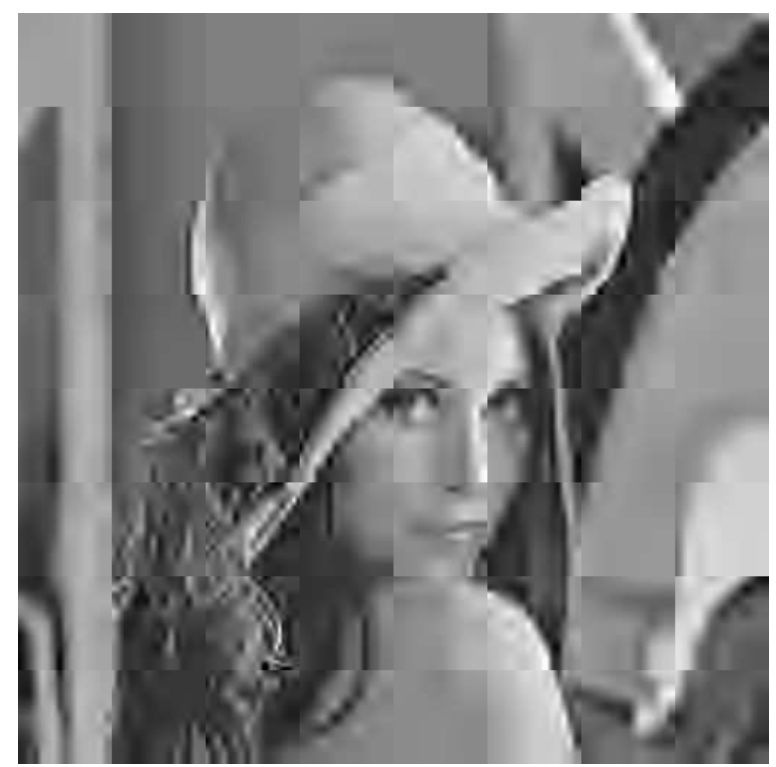

(b) JPEG2000 with a tile size $64 \times 64$ pixels. Bit rate is $0.1 b p p$. $P S N R=25.12 d B$

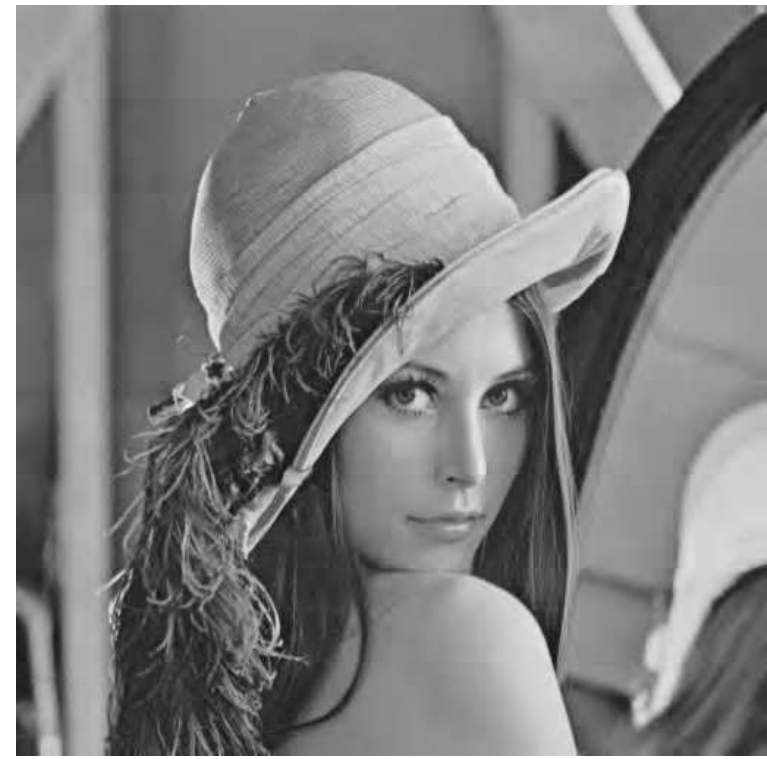

(d) JPEG2000 with a tile size $64 \times 64$ pixels. Bit rate is $0.5 b p p$. $P S N R=35.67 d B$

Fig. 5. Compression of Lena image.

experimental procedure for estimating the parameters of the computation energy model and the simulation details regarding the distribution of the wavelet transform tasks among the nodes.

1) Simulation setup: For each network topology, the nodes organize into clusters according to a clustering algorithm ${ }^{3}$ [42]. During each of the two sets of simulations reported, the values of the parameters of our proposed distributed image

\footnotetext{
${ }^{3}$ The choice of clustering algorithm is arbitrary and is done simply for simulation purpose. We leave the examination of using other clustering algorithms in our proposed distributed image compression for future research.
}

compression are chosen as follows. Every node is assumed to have an image Lena of size $512 \times 512$ pixels with 8 bits per pixel (bpp). We fix the number of nodes running distributed wavelet transform within each wavelet decomposition level to be 4 . Let $L$ denote the desired decomposition level of wavelet transform. The maximum value of $L$ for every image is chosen to be $5^{4}$. For simplicity, the desired image quality $Q$ is given in terms of the bit rate of final compressed image for this set

${ }^{4}$ It is chosen according to the size of the test image and to be compatible with centralized image compression scheme. The effect of decomposition level on the image quality and energy consumption is out of the scope of this paper and can be found in [18]. 
of simulations. Similar trends are observed for other values not reported here (for space considerations).

While our above approaches divide the computation workload of a single JPEG2000 image compression among multiple nodes in each wavelet decomposition level, they only consider the response of a single query (defined earlier as "session") between a source and a destination. If the processing node sets were chosen a priori and fixed throughout the system lifetime, it is easy to see that those unlucky nodes chosen to be processing nodes would run out of their energy quickly. Thus, in the simulation, we include randomized rotation of the processing node set such that it rotates among the various nodes among sessions in order to not drain the battery of a single node. In this way nodes will evenly share the workload of the network.

2) Computation and communication energy parameters: The values of the parameters of the computation energy model (3) and (4) are estimated as follows. We have employed JouleTrack [27] to estimate the energy consumption for an existing JPEG2000 $\operatorname{coder}^{5}$ [41]. The experiment data in terms of energy expended by a StrongARM SA -1100 processor at $206 \mathrm{Mhz}$ is measured when running JPEG2000 image compression algorithm on test image Lena (512x512). From the experiment, the value of $\gamma$ in (3) is estimated to be $220 \times 10^{-9}$ Joule/bit and the value of $\delta$ in (4) is estimated to be $20 \times 10^{-9}$ Joule/bit.

The values of the parameters of the wireless communication energy model (1) and (2) are the typical values $\epsilon_{a}=100 \times$ $10^{-12}$ Joule $/ \mathrm{bit} / \mathrm{m}^{2}$ and $\epsilon_{e}=50 \times 10^{-9}$ Joule $/ \mathrm{bit}$ as for example in [42]. The communication range of a node $d$ and is chosen to be $10 \mathrm{~m}$ for all nodes in this study, and $\alpha=2$.

3) Distributed operation in small networks: In the case of distributed image compression, if there are no sufficient nodes to organize into the required number of clusters, (for example, the distance between source and destination is not large enough) the last node(s) on the path to the destination may have to perform multiple wavelet transform operations on the remaining data until the required decomposition level is reached. We call this the "last-hop overload." This may potentially cause the nodes closer to the destination to deplete their energies faster. For example, $c_{4}$ will compute the remaining two wavelet transform levels on corresponding data in Fig. 6 before sending the final compressed image to the destination, if the required wavelet decomposition level was 5 .

We believe that for most common scenarios (except maybe some pathological cases), the last-hop overload will not have a significant effect on the system lifetime. There are two intuitive reasons for this:

1) The computational cost at the higher levels of wavelet decomposition is small compared to the lower levels of decomposition (e.g., the computational cost at the

\footnotetext{
${ }^{5}$ All options of this JPEG2000 coder are the default values except decomposition level that is chosen to be 1 since $\gamma$ is the energy dissipated for one level wavelet image compression per bit. Recall that $\gamma$ counts the energy consumed by wavelet transform, quantization and entropy coding.
}

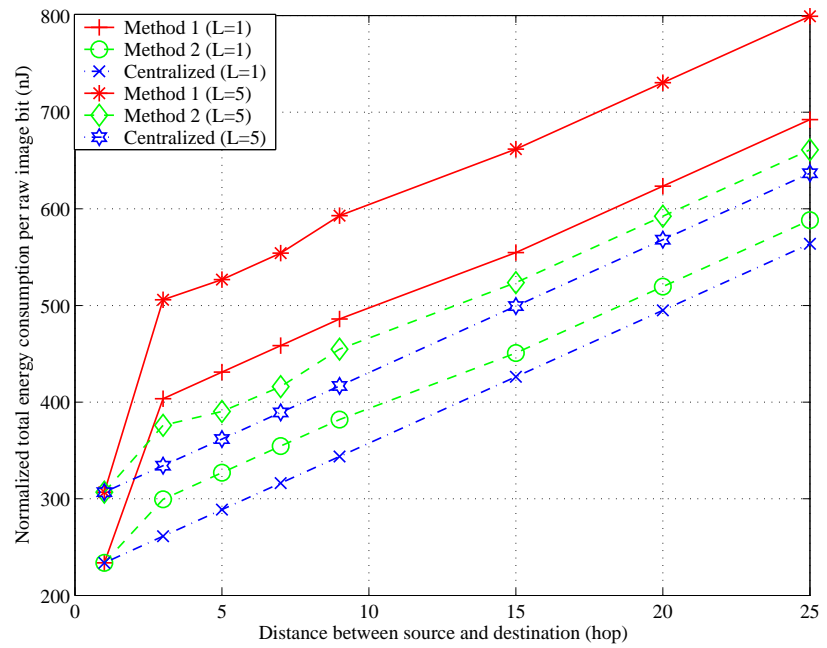

Fig. 7. Normalized total energy dissipation per raw image bit versus distance between source and destination for different desired decomposition level $L$. $Q=1 b p p$.

$i+1^{\text {th }}$ level of decomposition is only $1 / 4$ of $i^{\text {th }}$ level in the case of octave-band decomposition).

2) For each session, since the data closer to the destination are in a more compressed form than those further upstream (towards the source), the communication cost of the node(s) closer to the destination is smaller than the communication cost of its previous node on the path.

The required number of wavelet decomposition levels in practice is typically small (e.g. the default and recommended value for JPEG2000 implementation in [41] is 5. Most sensor network applications are assumed to involve a reasonably large number of sensors and the number of hops between the source and destination (sink) is most likely large enough to have at least 5 clusters for one wavelet decomposition level per cluster. For the reasons above, the nodes closest to the sink are less likely to be overburdened with computational requirements.

\section{B. Results}

1) Total Energy Consumption: We consider a network with 500 nodes randomly placed in an area of $125 \mathrm{~m} \times 125 \mathrm{~m}$. The node closest to the center is chosen to be the source. The destination nodes are chosen with varied distance from 1 to 25 hops between the source and the destination. The total energy dissipation of a session, which includes communication among nodes and distributed image compression, is measured for different source destination distance.

The comparison between the total energy dissipation of the three schemes (centralized, distributed Method 1 and distributed Method 2) is shown in Fig. 7 and Fig. 8. We examine the energy consumption with respect to the distance between source and destination for different wavelet decomposition level $L$ and image quality $Q$. It is observed in these figures that the total energy consumption of Method 1 is much higher than the other two under all circumstance. The total energy dissipation for Method 2 is slightly higher than centralized 


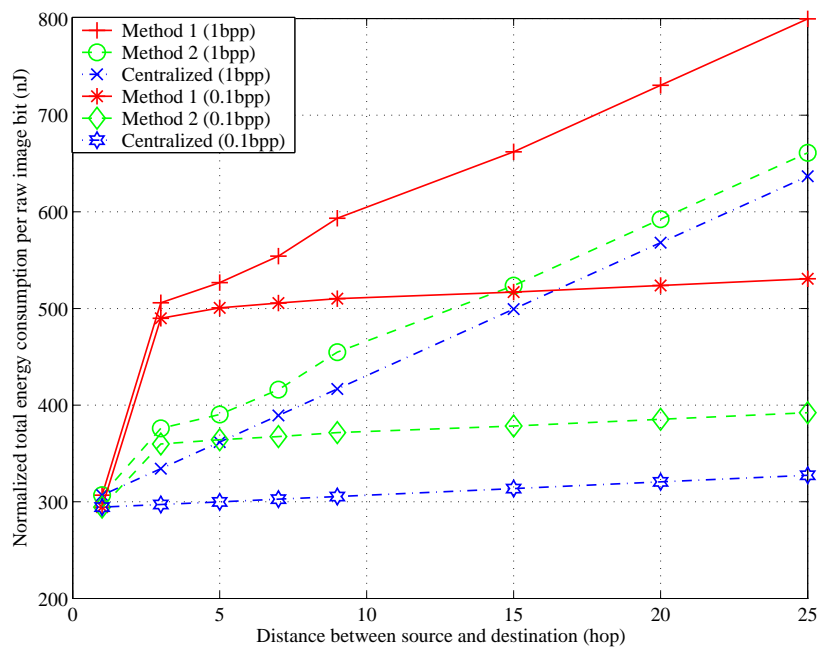

Fig. 8. Normalized total energy dissipation per raw image bit versus distance between source and destination for different desired image quality $Q$. $L=5$.

image compression (where only source node runs JPEG2000 image compression algorithm), particularly for moderate or high bit rate quality requirement. However, in terms of system lifetime, the distributed approach of Method 2 has better performance, as described in the next simulation results.

2) System Lifetime: In this section, we only compare Method 2 (and not Method 1) with the centralized image compression since it is shown in the previous section that it performs much better than Method 1 in terms of total energy consumption.

We consider five connected networks of size $N=25,50$, 100,200 or 500 nodes. Nodes are randomly placed in a square area with average node degree (i.e. number of neighboring nodes) to be 10 . The area size is computed to keep the network asymptotically connected ${ }^{6}$. We choose the node which is the closest to the center of the field as the source. For every session, the destination is randomly chosen. Each node is initially given 1 Joule of energy. The simulation is stopped if any node in the network depletes its energy. The system lifetime in terms of number of sessions is measured for both distributed and centralized image compression.

The comparison between the system lifetime achieved by using our approach against a centralized scheme is shown in Fig. 9 and Fig. 10. Very similar trends are observed for different values of $Q$ and $L$ in these figures. In the case of conventional centralized image compression, the source node "dies" quickly since it consumes power at a very high rate compared to other nodes in the network. The system lifetime of centralized image compression is almost constant

${ }^{6}$ Let $L$ denote the side length of the square. Let $g$ denote the average degree of a node. From [43], we know that this average node degree should be $\Theta(\log N)$ to keep the network asymptotically connected. The side length $L$ of every network size is calculated from

$$
L=\lfloor d \sqrt{N \pi / g}\rfloor
$$

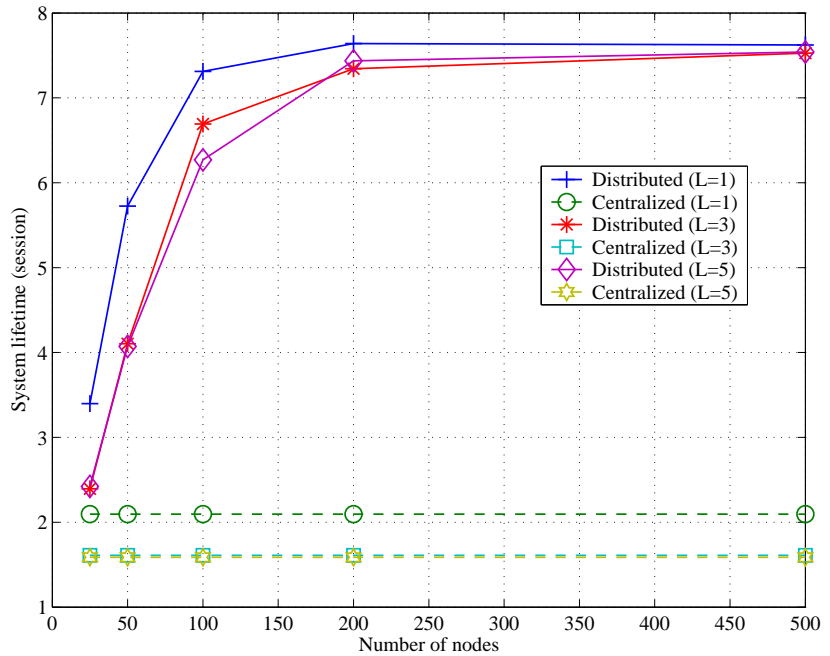

Fig. 9. System lifetime comparison: distributed versus centralized for different desired decomposition level $L . Q=1 b p p$.

for different network sizes. On the other hand, the results of Method 2 of the distributed approach result in a much longer lifetime since it divides the computation workload uniformly (statistically speaking) among all the nodes in the network. The results show that considerably longer system lifetime (e.g., up to 4 times with large network size) can be achieved when using distributed image compression (Method 2) algorithm.

It is observed that the system lifetime of distributed image compression scheme (Method 2) increases as the network size increases for small to moderate network size, then almost becomes flat for large network size. We provide an intuitive explanation for this observation. Consider first the case of very small network size (in terms of number of nodes). In this case, the source and destination are very close to each other as is the case in a small network, our proposed distributed scheme can not be applied because of insufficient nodes and it defaults to a variation of the centralized scheme. In this case, the source nodes perform most of the processing and tend to fail first, limiting the lifetime of the network.

As the network size increases, it becomes more likely that there are sufficient nodes between the source and destination to implement the proposed distributed scheme and hence extend the network lifetime. However, observe that the first level wavelet transform is the most consuming in terms of computation energy, and hence the processing nodes in the cluster closest to the source are more likely to deplete their energies first. Since this number is fixed in our simulations, the lifetime of the network remains constant as the network size grows to very large sizes. Increasing the number of processing nodes in each cluster would increase the network lifetime, but the overall trend (saturation for a specific number of processing nodes) will still be observed (simulations not reported here for this case since the curves are similar to the reported results). 


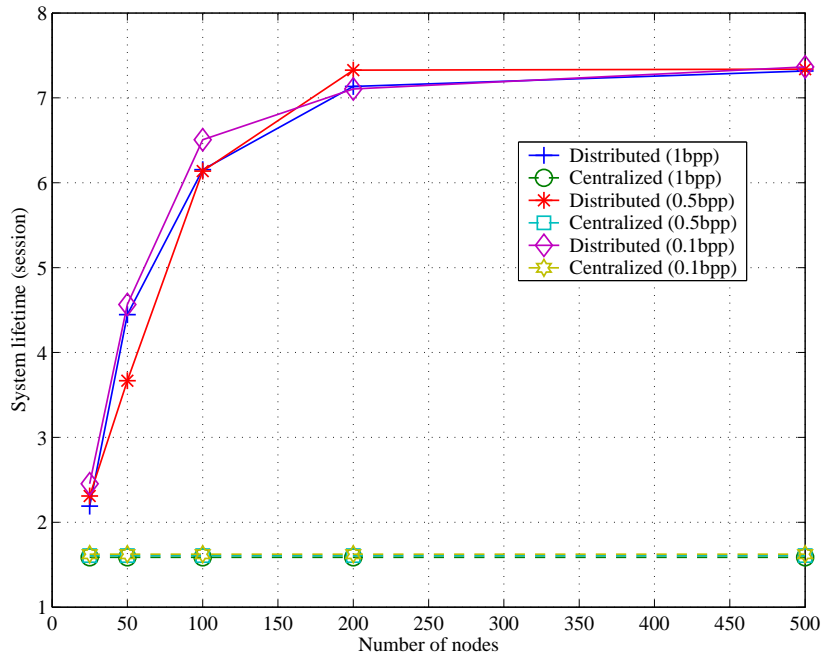

Fig. 10. System lifetime comparison: distributed versus centralized for different desired image quality $Q . L=5$.

\section{CONCLUSION AND FuturE WORK}

In this paper, we studied the problem of energy efficient image encoding/transmission in multi-hop wireless networks. The design and evaluation of two design alternatives for distributed image compression scheme is presented. We use a combination of tiling of images, and load balancing by nodes rotation to achieve much longer system lifetime compared to the centralized image compression. The proposed scheme is simple and easy to implement. Performance evaluation shows that this distributed scheme can have significantly longer system lifetime while satisfying the performance constraint in terms of a target image quality compared to a centralized approach.

This work provides an important proof of concept that shows the benefits and feasibility of distributed image compression. Several aspects of future research are the impact of wireless link errors, and the use of multi-path routing to enhance the performance of distributed image compression. In the future, we plan to validate our approach on a sensor network testbed.

\section{ACKNOWLEDGMENT}

The authors would like to thank Ms. Ying Liu, for many useful discussion on the wavelet transform, Prof. William A. Pearlman for valuable input and discussion, and the anonymous reviewers, for multiple suggestions to improve the quality of presentation.

\section{REFERENCES}

[1] "Digital pixel sensor," 2004. [Online]. Available: http://www-isl stanford.edu/ abbas/group/

[2] A. E. Gamal, "Trends in CMOS image sensor technology and design," in Proceedings of IEEE International Electron Devices Meeting, San Francisco, CA, December 2002.
[3] D. Estrin, "Sensor network research: Emerging challenges for architecture, systems, and languages," in Proceedings of the 10th International Conference on Architectural Support for Programming Languages and Operating Systems (ASPLOS-X), ser. ACM SIGPLAN notices, C. Norris and J. J. B. Fenwick, Eds., vol. 37, 10. New York: ACM Press, October 5-9 2002, pp. 1-4.

[4] A. E. Gamal, "Collaborative visual sensor networks," 2004. [Online] Available: http://mediax.stanford.edu/projects/cvsn.html

[5] G. Zaruba and S. Das, Off-the-shelf enablers of ad hoc networks. New York: IEEE Press Wiley, 2003.

[6] "SmartDust," 2003. [Online]. Available: http://robotics.eecs.berkeley. edu/ $\sim$ pister/SmartDust/

[7] C. Intanagonwiwat, R. Govindan, and D. Estrin, "Directed diffusion: a scalable and robust communication paradigm for sensor networks," in Proceedings of the sixth annual international conference on Mobile computing and networking. ACM Press, 2000, pp. 56-67.

[8] W. Zhang, Z. Deng, G. Wang, L. Wittenburg, and Z. Xing, "Distributed problem solving in sensor networks," in Proceedings of the first international joint conference on Autonomous agents and multiagent systems. ACM Press, 2002, pp. 988-989.

[9] A. Wang and A. Chandrakasan, "Energy efficient system partitioning for distributed wireless sensor networks," in Proceedings of the International Conference on Acoustics, Speech, and Signal Processing (ICASSP-2001), Salt Lake City, Utah, May 7-11 2001.

[10] S. S. Pradhan, J. Kusuma, and K. Ramchandran, "Distributed compression in a dense microsensor network," IEEE Signal Processing Magazine, vol. 19, no. 2, pp. 51 -60, March 2002.

[11] D. P. Bertsekas and J. N. Tsitsiklis, Parallel and distributed computation: numerical methods. Prentice-Hall, Inc., 1989.

[12] M. Martina, G. Masera, G. Piccinini, and M. Zamboni, "A VLSI architecture for IWT (integer wavelet transform)," in Proceedings of the 43rd IEEE Midwest Symposium on Circuits and Systems, vol. 3, 2000, pp. $1174-1177$.

[13] J. Ritter and P. Molitor, "A partitioned wavelet-based approach for image compression using FPGA's," in Proceedings of the IEEE Custom Integrated Circuits Conference, 2000, pp. 547 - 550.

[14] K. Haapala, P. Kolinummi, T. Hamalainen, and J. Saarinen, "Parallel DSP implementation of wavelet transform in image compression," in Proceedings of The 2000 IEEE International Symposium on Circuits and Systems, vol. 5, Geneva, 2000, pp. $89-92$

[15] D. Chaver, M. Prieto, L. Pinuel, and F. Tirado, "Parallel wavelet transform for large scale image processing," in Proceedings of International Parallel and Distributed Processing Symposium, IPDPS, 2002, pp. 4-9.

[16] P. Meerwald, R. Norcen, and A. Uhl, "Parallel JPEG2000 image coding on multiprocessors," in Proceedings of the International Parallel and Distributed Processing Symposium 2002, 2002, pp. 2-7.

[17] C. F. Chiasserini and R. R. Rao, "On the concept of distributed digital signal processing in wireless sensor networks," in Proceedings of MILCOM, vol. 1, 2002, pp. 260-264.

[18] H. Wu and A. A. Abouzeid, "Power aware image transmission in energy constrained wireless networks," 2004, in the IEEE symposium on Computers and Communications (ISCC). [Online]. Available: http://www.rpi.edu/ $\sim_{\text {wuhm/research/iscc04.pdf }}$

[19] D. Devaguptapu and B. Krishnamachari, "Applications of localized image processing techniques in wireless sensor networks," in SPIE's 17th Annual International Symposium on Aerospace/Defense Sensing, Simulation, and Controls, (Aerosense '03), Orlando, Florida, April 2003.

[20] D. Ganesan, B. Greenstein, D. Perelyubskiy, D. Estrin, and J. Heidemann, "An evaluation of multi-resolution storage for sensor networks," in Proceedings of the first international conference on Embedded networked sensor systems. ACM Press, 2003, pp. 89-102.

[21] S. D. Servetto, "Sensing lena-massively distributed compression of sensor images," in Proceedings of the IEEE International Conference on Image Processing (ICIP) (special session on "Distributed Source Coding”), Barcelona, Spain, September 2003.

[22] E. Woodrow and W. Heinzelman, "SPIN-IT: A data centric routing protocol for image retrieval in wireless networks," in International Conference on Image Processing (ICIP '02), September 2002.

[23] J. Pan, Y. T. Hou, L. Cai, Y. Shi, and S. X. Shen, "Topology control for wireless sensor networks," in Proceedings of the 9th annual international conference on Mobile computing and networking. ACM Press, 2003, pp. 286-299.

[24] S. Bandyopadhyay and E. J. Coyle, "An energy-efficient hierarchical clustering algorithm for wireless sensor networks," in INFOCOM, 2003. 
[25] E. Shih, S.-H. Cho, N. Ickes, R. Min, A. Sinha, A. Wang, and A. Chandrakasan, "Physical layer driven protocol and algorithm design for energy-efficient wireless sensor networks," in Proceedings of the Seventh Annual International Conference on Mobile Computing and Networking (MOBICOM-01). ACM Press, July 16-21 2001, pp. 272 287.

[26] T. S. Rappaport, Wireless Communications: Principles and Practice. IEEE Press, 1996.

[27] A. Sinha and A. Chandrakasan, "JouleTrack - a web based tool for software energy profiling," in Design Automation Conference, 2001, pp. 220-225. [Online]. Available: http://www-mtl.mit.edu/research/anantha/ jouletrack/JouleTrack/index.html

[28] G. K. Wallace, "The JPEG still picture compression standard," Communications of the ACM, vol. 34, no. 4, pp. 30-44, 1991.

[29] S. G. Mallat, "A theory for multiresolution signal decomposition: The wavelet representation," IEEE Transactions on Pattern Analysis and Machine Intelligence, vol. 11, no. 7, pp. 674-693, July 1989.

[30] C. A. Christopoulos, T. Ebrahimi, and A. N. Skodras, "JPEG2000: the new still picture compression standard," in Proceedings of the 2000 ACM workshops on Multimedia. Los Angeles, California, United States: ACM Press, 2000, pp. 45-49.

[31] Y. Chan, Wavelet Basics. Norwell, MA: Kluwer Academic Publishers, 1995.

[32] D.-G. Lee and S. Dey, "Adaptive and energy efficient wavelet image compression for mobile multimedia data services," in IEEE Intl. Conference on Communications (ICC), New York, 2002.

[33] J. K. Martin Vetterli, Wavelets and Subband Coding. Englewood Cliffs, NJ: Prentice Hall, 1995.

[34] A. Ortega, "Optimal rate allocation under multiple rate constraints," in Proceedings of Data Compression Conference (DCC), Snowbird, UT, March 1996.

[35] J. Storer, Data Compression. Rockville, MD: Computer Science Press, 1988.

[36] F. Marino, V. Piuri, and E. J. Swartzlander, "A parallel implementation of the 2-D discrete wavelet transform without interprocessor communications," IEEE Transactions on Signal Processing, vol. 47, no. 11, pp. 3179 - 3184, November 1999.

[37] A. Nosratinia, "Post processing of JPEG-2000 images to remove compression artifacts," IEEE Signal Processing Letters, vol. 10, no. 10, pp. 296-299, October 2003.

[38] D. S. Taubman and M. W. Marcellin, JPEG2000, Image Compression Fundamentals, Standards and Practice. Kluwer Academic Publishers, 2002.

[39] D. S. Taubman, "High performance scalable image compression with ebcot," IEEE Trans. Image Processing, vol. 9, pp. 1158-1170, July 2000.

[40] K. C. Barr and K. Asanovic, "Energy aware lossless data compression," in The First International Conference on Mobile Systems, Applications, and Services, San Francisco, CA, May 2003.

[41] M. D. Adams, "The JasPer project home page," 2003. [Online]. Available: http://www.ece.uvic.ca/ mdadams/jasper/

[42] A. C. Wendi Rabiner Heinzelman and H. Balakrishnan, "Energyefficient communication protocol for wireless microsensor networks," in Proceedings of the Hawaii International Conference on System Sciences (HICSS), Jan. 4-7 2000.

[43] F. Xue and P. R. Kumar, "The number of neighbors needed for connectivity of wireless networks," Wireless Networks, vol. 10, no. 2, pp. 169-181, 2004.

[44] "ns-Network Simulator," http://www.isi.edu/nsnam/ns/, 1998. 